\title{
Effectiveness of a Patient-Specific Immobilization and Positioning System to Limit Interfractional Translation and Rotation Setup Errors in Radiotherapy of Prostate Cancers
}

\author{
Gilbert Law, Ronnie Leung, Frankle Lee, Hollis Luk, Ka Chai Lee, Frank Wong, \\ Matthew Wong, Steven Cheung, Venus Lee, Wing Ho Mui, Mark Chan* \\ Department of Clinical Oncology, Tuen Mun Hospital, Hong Kong, China \\ Email: *Mark.Chan@uhs.nhs.uk
}

Received 11 June 2016; accepted 19 August 2016; published 22 August 2016

Copyright (C) 2016 by authors and Scientific Research Publishing Inc.

This work is licensed under the Creative Commons Attribution International License (CC BY).

http://creativecommons.org/licenses/by/4.0/

(c) (i) Open Access

\section{Abstract}

Objective: To evaluate the effectiveness of a patient-specific immobilization and positioning device in prostate radiotherapy. Methods: Eighty patients were immobilized and positioned by a patientspecific device. Prostate translations and rotations were estimated from daily cone beam computed tomography scans using a contour-based approach assisted by auto-registration and quantified by the group mean GM, systematic $\Sigma$ and random $\sigma^{\prime}$ errors. Dosimetric impacts of residual prostate rotations where the translation errors were corrected were evaluated by robustness plan analysis. Results: Using the patient-specific immobilization alone without online image-guidance, the GM, $\Sigma$ and $\sigma^{\prime}$ of the prostate translations were $0.8,1.7$, and $1.5 \mathrm{~mm}$ (left-right; LR), $0.8,2.1$, and $1.9 \mathrm{~mm}$ (superior-inferior; SI), and $0.5,1.7$ and $1.5 \mathrm{~mm}$ (anterior-posterior; AP), while for the prostate rotations they were $0.0^{\circ}, 0.6^{\circ}$, and $0.7^{\circ}$ (pitch), $0.2^{\circ}, 0.5^{\circ}$, and $0.6^{\circ}$ (roll), and $0.2^{\circ}, 0.5^{\circ}$, and $0.6^{\circ}$ (yaw). The resulting van Herk's margin was 5.8 (LR), 7.3 (SI) and 5.8 (AP) $\mathrm{mm}$. With adaptive online imageguidance based on estimates from the first 5 fractions, $\Sigma$ were reduced by $0.7-1.2 \mathrm{~mm}$ for the prostate translations, resulting in a margin reduction by $2-3.5 \mathrm{~mm}$. Changes of $\Sigma$ and $\sigma^{\prime}$ in the prostate rotations were insignificant regardless of translation corrections. Dosimetric impacts of residual rotation errors were negligible if a $2 \mathrm{~mm}$ margin was applied. Conclusions: Our patientspecific immobilization system can effectively limit the prostate translations and rotations, which is important without 6D treatment couches or using ultrasound image-guidance without rotational corrections. 


\section{Keywords}

\section{Immobilization, Prostate, CBCT, Margin, Interfractional Motion}

\section{Introduction}

Emerging data from retrospective studies and a few randomized trials have suggested a clear dose-response relationship between biochemical relapse rate and radiation dose in radiotherapy for prostate cancers [1]-[3]. Madsen et al. have reported actuarial 48-month biochemical freedom from relapse of 70\% in a Phase I/II trial of stereotatic prostate radiotherapy using $33.5 \mathrm{~Gy}$ in five fractions [4]. In a randomized trial of $70 \mathrm{~Gy}$ vs. $80 \mathrm{~Gy}$ in conventional 2-Gy fractions, Beckendorf et al. have reported that 5-year biochemical relapse rate was $11 \%$ less in the 80 Gy arm [1].

Accurate delivery of high radiation doses to the prostate target necessitates image-guidance (IG) to reduce the geometric uncertainty and hence the treatment margin to avoid significant rectal and urinary toxicities [5]. Onboard kilovoltage - $(\mathrm{kV})$ and megavoltage - (MV) cone beam computed tomography (CBCT) [6], [7], 2D/3D registrations of implanted markers based on $\mathrm{kV}$ stereoscopic imaging [8], [9] and electromagnetic tracking [10], and ultrasound imaging [11], [12] are current IG options for management of inter- and intrafractional variability of the target position. Most of the IG systems, except the BAT ${ }^{\mathrm{TM}}$ (B-Mode Acquisition and Targeting system; NOMOS Corp, Sewickley, PA, USA) and the Clarity (Elekta AB, Sweden) ultrasound imaging systems [11], [12], provide the solution of target shift corrections through three translational and three rotational errors of the target position through rigid body image registrations based on bony or soft-tissue anatomy, or fiducial markers. However, correction strategies in common clinical practice deal with the target shift mainly by management of the translational errors because standard treatment couches can only be rotated around one axis that is often ignored if the associated rotational error is less than some threshold (e.g., $2^{\circ}-3^{\circ}$ ). Several groups have derived systems of coordinate transformations to adjust the angles of gantry and collimator for approximate corrections in the left-right (LR) rotation errors [13], and in additional to couch angle adjustment for corrections in all three rotational errors for IMRT treatment [14], [15]. For VMAT treatment, the coordinate transformations must be done for a large number of treatment fields, called segments, at a series of consecutive gantry angles (or control points), typically $360^{\circ}$ for a full-arc VMAT. Most importantly, even a solution can be obtained to correct for every treatment field angle in the arc, the collimator and couch may have to be rotated continuously from angle to angle during the arc delivery. Such continuous motion represents a hardware constraint for all linac systems. Hence, the residual rotation errors must be explicitly incorporated into the planning target volume (PTV).

Lips et al. have reported that LR rotation was dominant in radiotherapy for prostate cancers and was associated with systematic and random errors of $6.3^{\circ}$ and $4.9^{\circ}$, respectively [16]. Strong correlation in the anteriorposterior and superior-inferior prostate motion was observed from real-time tracking of intrafractional prostate suggesting a longitudinal oblique motion of the prostate [10]. Duetschmann et al. also reported a $5 \%$ chance of observing rotations $>15^{\circ}$ which alone requires $8 \mathrm{~mm}$ safety margin to compensate [15]. Otherwise, $12 \%$ underdose in portions of seminal vesicles and prostate apex was expected when only translation errors were corrected and a $6 \mathrm{~mm}$ margin was applied. Similar results were reported by Shang et al., showing a decrease of 3.6\% $\pm 9.0 \%$ in dose to $99 \%$ of the clinical target volume (CTV) if only the translation errors were corrected [17]. This highlights the importance of effective immobilization to control the rotation errors if they are not compensated for in the daily on-line correction protocol.

The impact of different immobilization systems on setup errors has been analyzed in a few studies based on $\mathrm{MV} / \mathrm{kV}$ port films and repeated simulation CT in terms of the translational errors [18]-[20]. At our institute, patients who underwent conventional 2 Gy-fractions radiotherapy were immobilized by means of an in-house immobilization body frame that has several laser indexers to assist the adjustment of the overall translation and rotation of the patient. We have reported the early clinical outcome for 20 patients treated with IMRT using such immobilization system [21]. This study aimed to evaluate the effectiveness and practicability of this immobilization device by retrospective analysis of both the translational and rotational errors obtained from daily CBCT guidance. Safety margins for different setup correction strategies combining with such immobilization device were calculated. An additional group of patients were subsequently re-planned to validate the calculated safety margins by robustness 
plan analysis to guide the setup of correction protocol-specific safety margin at our institution.

\section{Methods and Materials}

\subsection{Patients and Treatment Protocol}

From 2011 to 2014, 80 patients treated to 72 to 76 Gy to prostate were selected for this IRB approved retrospective study. Twenty patients were randomly sampled from each year, involving eighteen radiotherapists in the treatment setup. The treatment technique changed from 5-fields intensity-modulated radiotherapy to volumetric-modulated arc radiotherapy (VMAT) since 2012.

\section{Patient Immobilization and CT Simulation}

All patients were comfortably immobilized by a customized alpha cradle inside the total body frame (TBF) that has a detachable positioning arc with three laser pointers (Figure 1). The longitudinal (in the superior-inferior direction) position of the positioning arc was adjusted so that the three indexing lasers pointed at about the level of the prostate and were marked (Figure 1(b)). This longitudinal position of the positioning arc (y-axis coordinate) was determined on the longitudinal scale and was used to set up the patient in the superior-inferior (SI) direction for each fraction. Additional skin marks were made on the patient's ankles, which correlated to a laser attached to the frame inferiorly (Figure 1(c)). Together, the laser indexing system aimed to provide control of the overall rotation of the patient's pelvis and femoral heads. All coordinates were recorded on a CT simulation sheet.

During the CT simulation, the positioning arc was removed from the TBF. The treatment isocenter shift was determined based on the longitudinal (y-axis), vertical (z-axis), and lateral (x-axis) coordinates labeled on the TBF (Figures 1(b)-(d)).

\subsection{Treatment Setup and Image-Guidance Correction Protocol}

For treatment setup, the treatment position of the patient was first adjusted until the skin marks over the pelvis region and the ankle aligned with the laser pointers. Next, the immobilized patient inside the body frame was aligned to the simulation isocenter according to the longitudinal, vertical and lateral coordinate recorded CT simulation sheet and eventually aligned to treatment isocenter according to the shifts during treatment planning.
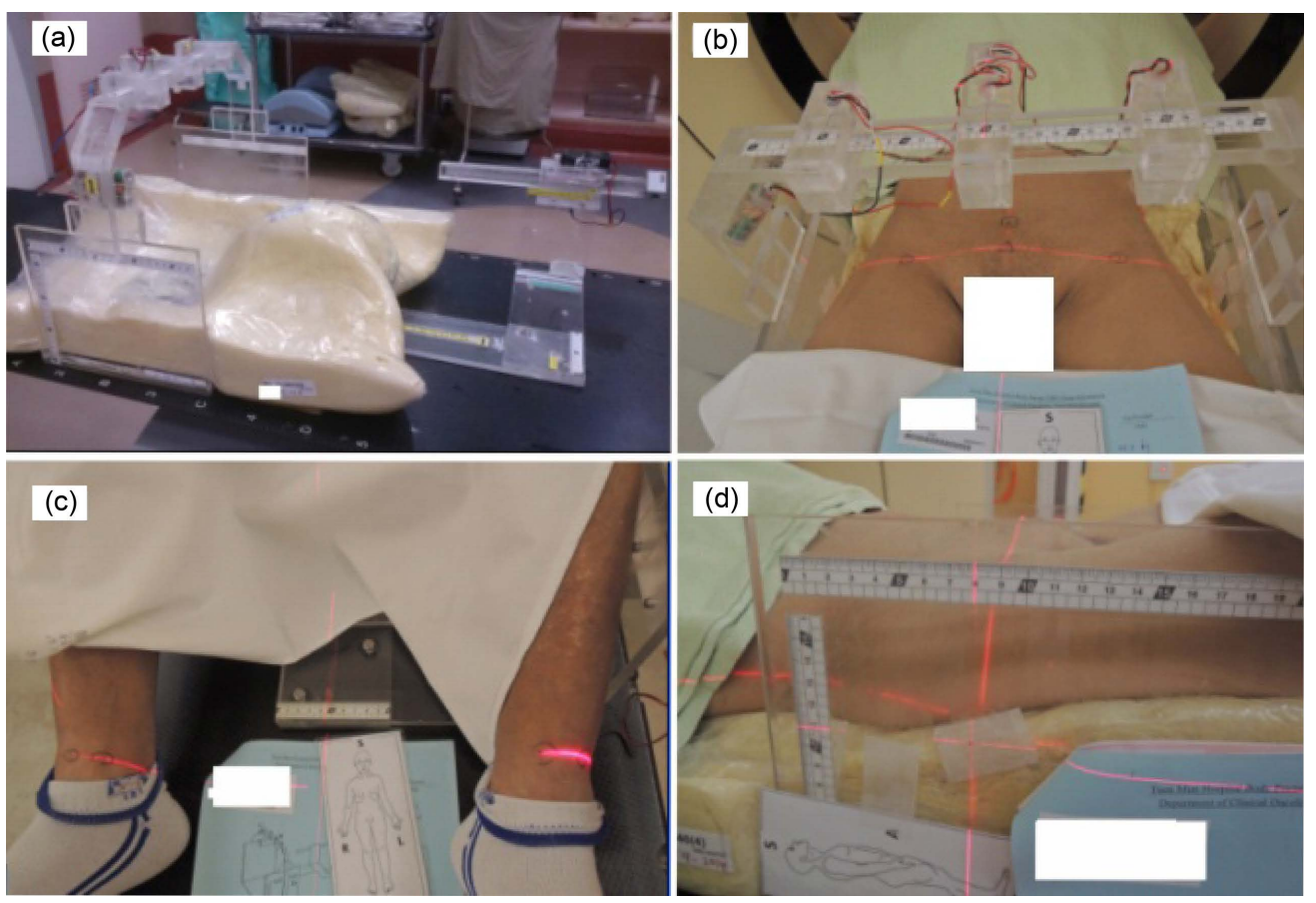

Figure 1. Customized total body frame (TBF) with alpha cradle. 
Pre-treatment CBCT was acquired at each fraction with the x-ray volume imaging (XVI) system R4.5 (Elekta Oncology Systems, Crawley, UK). Each 3D-CBCT scan acquired 660 projection images at 5 frames per second over $360^{\circ}$ in 2 minutes. After the CBCT acquisition, a soft-tissue registration was performed by a user-defined 3D region-of-interest (ROI) that included the regions of the whole prostate, seminal vesicle and anterior portion of the rectum while excluding the bony structure (e.g., the femoral heads and the pelvic bones), yielding three translational ( $T_{x}$ : left-right (LR), $T_{y}$ : superior-inferior (SI), and $T_{z}$ : anterior-posterior (AP)) and three rotational errors $\left(R_{x}\right.$ : pitch, $R_{y}$ : roll, and $R_{z}$ : yaw) relative to the TBF system. Prostate translations in the left, superior, and posterior direction are defined as positive while clockwise rotations from the left, superior, and posterior along the AP, LR, and SI axes are defined as positive. Subsequently, each contoured prostate with or without the seminal vesicles was matched to the planning CT scan to obtain the final translations and rotations. For actual treatments, automatic couch movement was made to correct only the translation errors if the rotation error in each rotation axis was $<2^{\circ}$. Otherwise, the patient was repositioned, and a second CBCT was performed to confirm that the rotation errors were $<2^{\circ}$.

For each patient $i$, the mean $(\mu)$ and standard deviation $(\sigma)$ of the variability of prostate position were determined for all fractions $n=1 \ldots 36$ or $38\left(\mu_{i, n}\right.$ and $\left.\sigma_{i, n}\right)$, and for the first $k=5$ fractions $\left(\mu_{i, k}, \sigma_{i, k}\right)$. If another CBCTs were acquired after patient repositioning because of excessive rotation errors, only the translational and rotational errors in the first CBCT were analyzed. Our clinical correction strategy employed $\mu_{i, k}$ as an estimator of the mean position of the prostate for correction of the remaining $n-k$ fractions. The residual variability in terms of $\mu_{i, n-k}$, and $\sigma_{i, n-k}$ were used to estimate the PTV margin of the adaptive treatment protocol by online correction for the first 5 fractions and offline thereafter. Population statistics about the interfractional variability of prostate position were quantified in terms of the group mean GM (i.e., mean of $\mu_{i, n}$ ), the systematic error $\Sigma$ and the random error $\sigma^{\prime}$ for the entire treatment using $\mu_{i, n}$ and $\sigma_{i, n}$, and similarly for the first $k=5$ fractions using $\mu_{i, k}$ and $\sigma_{i, k}$, and for the remainder of $n-k$ fractions using $\mu_{i, n-k}$, and $\sigma_{i, n-k}$ after online correction for the first 5 fractions.

\subsection{Estimation of CTV-to-PTV Margin}

Despite the on-line IG correction strategy, we employed a relatively conservative $10 \mathrm{~mm}$ PTV margin except at the posterior side of the CTV with a $5 \mathrm{~mm}$ margin to spare the rectum in all clinical treatments. This margin was not optimized and was known to result in an increased amount of doses to the normal tissues, particularly the rectum, bladder. With the daily IG data analyzed in this study, we sought to determine retrospectively the appropriate PTV margin assuming 1) non-IG setup (i.e., using the TBF alone), 2) online adaptive correction (i.e., using the TBF with online IG correction for $\mu_{i, k}$ and offline thereafter), and 3) on-line correction setup (i.e., daily CBCT together with the TBF). The CTV-to-PTV margin $M$ was approximated by the van Herk's margin recipe [22]: $M=2.5 \Sigma+0.7 \sigma^{\prime}$, assuming that $95 \%$ of the population received $95 \%$ of the prescription dose to the target. Note that we did not compute for the scenario of online adaptive correction the combined errors from the first $k$ fractions prior to correction (i.e., $\Sigma_{k}$ and $\sigma_{k}^{\prime}$ ) and the remainder $n-k$ (i.e., $\Sigma_{n-k}$ and $\sigma_{n-k}^{\prime}$ ) in the calculation of PTV because $\mu_{i, k}$ and $\sigma_{i, k}$ (from $T_{x}, T_{y}$, and $T_{z}$ ) of individual patients were effectively eliminated [23]. Furthermore, the van Herk's margin recipe per se does not deal with uncertainty related to rotations, and a $2 \mathrm{~mm}$ PTV margin was assumed for on-line correction setup with the TBF.

\subsection{Simulation of Translational and Rotational Errors in Different Correction Strategies}

To determine the dosimetric impacts of setup errors, 5 other patients from 2014 were randomly sampled from our database and were re-planned by replacing the original PTV with margins calculated for different setup correction strategies, as described in the previous section. Dosimetric constraints per our institution's protocol included the prescription dose (76 Gy) coverage of the PTV > 95\% (V76 > 95\%) and minimum dose to the GTV > 76 Gy, rectum and bladder volumes receiving 65 Gy (V65) < 20\% and 25\%, and receiving 40 Gy (V40) < 45\% and $60 \%$, respectively.

These plans were then imported into CERR computational research platform [24] to perform plan robustness analysis by simulation of a large number of trial (300) based on the individual's systematic and random errors, with each trial representing the total dose delivery, i.e. all treatment fractions. The DVHs were calculated or the simulated doses for all the trials, and from these DVHs the mean and standard deviation were estimated for volume fractions corresponding to different dose bins. The expected DVH was then the DVH constructed from 
dose bins and the mean volume. It was used to validate the adequacy of the margins calculated for different treatment setup scenarios.

\subsection{Statistics}

For statistical tests of normality by the Kolmogorov-Smirnov normality test, one sample $t$-test, or Wilcoxon test for the difference of group mean from zero, and two sample paired $t$-test or Wilcoxon test for the differences of translation and rotation errors between setup correction strategies, the MATLAB Statistical Toolbox (Math Works Inc., Natick, MA, USA) was used. Statistical significance was considered with $p$-value $<0.05$.

\section{Results}

\subsection{Absolute Prostate Displacement}

A total of 2679 pre-treatment CBCT was analyzed. Figure 2 shows the cumulative probability distribution (CDF) of $\mu_{i, n}$ in $T_{x}, T_{y}$, and $T_{z}$ and in $R_{x}, R_{y}$, and $R_{z}$ for non-IG setup using TBF alone. In one patient, the mean $T_{y}$ error was found to be as large as $13.5 \mathrm{~mm}$ because the longitudinal coordinate of the body frame was misrecorded during CT simulation. The translation error was more frequent in the right (74.7\%), inferior (73.3\%), and anterior (65.3\%) directions although the magnitudes were of similar order in both directions (1.5 vs. $1.2 \mathrm{~mm}$ in left-right, 1.6 vs. $1.2 \mathrm{~mm}$ in superior-inferior, and $1.4 \mathrm{vs.} 1.3 \mathrm{~mm}$ in the anterior-posterior directions). Except for $R_{y}$ (roll), $R_{x}$ (pitch) and $R_{z}$ (yaw) rotations in either direction were equally probable within $2 \%$. The $95^{\text {th }}$ percentiles of the absolute $\mu_{i, n}$ were 3.6, 3.5, and $3.6 \mathrm{~mm}$ for $T_{x}, T_{y}$ and $T_{z}$, and $1.0^{\circ}$ for $R_{x}, R_{y}$, and $R_{z}$, respectively.
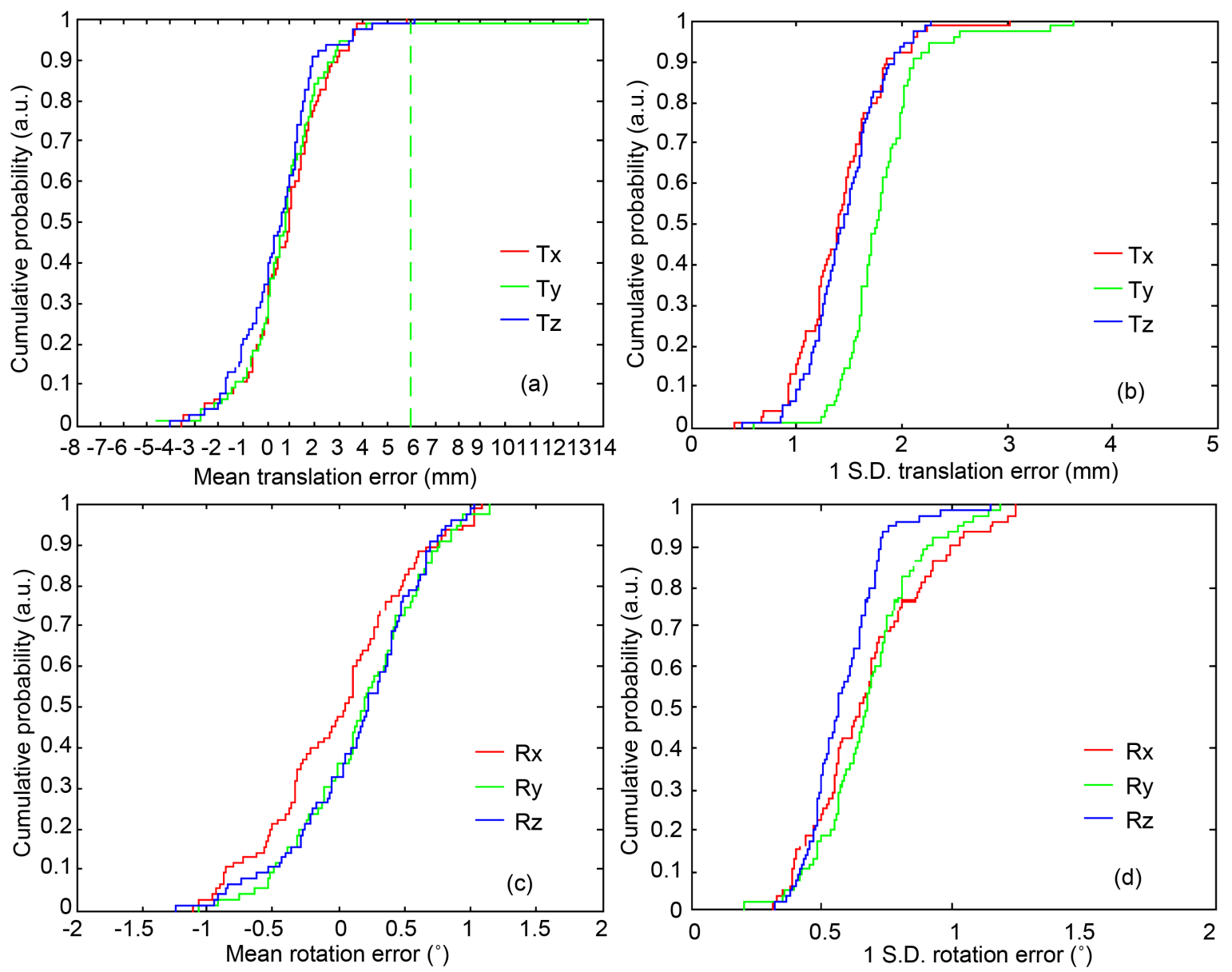

Figure 2. Cumulative probability curve of (a) mean translation error; (b) standard deviation of translation error; (c) mean rotational error; (d) standard deviation of rotational error, for non-IG setup with TBF. 
The cumulative distributions of $\mu_{i, n-k}$ in $T_{x}, T_{y}$, and $T_{z}$ and in $R_{x}, R_{y}$, and $R_{z}$ for the adaptive online correction setup with TBF were shown in Figure 3. After online correction for $\mu_{i, k}$, the $95^{\text {th }}$ percentiles of the absolute $\mu_{i, n-k}$ were found to be $1.2,1.6$ and $2.0 \mathrm{~mm}$ for $T_{x}, T_{y}$ and $T_{z}$, and $1.2^{\circ}, 0.9^{\circ}$ and $1.0^{\circ}$ for $R_{x}, R_{y}$ and $R_{z}$, respectively. Except in the left-right direction where the translational error was more frequent to the left (61.3\%), translations and rotations in all opposite direction shared more or less equal probability within $3 \%$, and differed by $0.1 \mathrm{~mm}$ and $0.1^{\circ}$, respectively.

\subsection{Population Statistics and PTV Margin}

The population statistics about the group means GM, systematic $\Sigma$ and random $\sigma^{\prime}$ errors for different setup strategies using the TBF were given in Table 1. Group means obtained from the first $k=5$ fractions and the total $n=36$ or 38 fractions using the TBF alone were found to differ significantly from zero $(p<0.05)$ for all translations and rotations except for $R_{x}$, whereas GM obtained from the $n-k$ fractions assuming online adaptive CBCT with TBF differed significantly from zero for the $R_{y}$ only.

Statistics of $\Sigma$ and $\sigma^{\prime}$ obtained with the first $k$ fractions and the total $n$ fractions using only the TBF are within $0.5 \mathrm{~mm}$ and $0.0^{\circ}$. $\Sigma$ and $\sigma^{\prime}$ errors for individual patients based on the first 5 fractions, and the remainders of the treatment after adaptive online correction, i.e., $\mu_{i, k}$ and $\mu_{i, n-k}$, and $\sigma_{i, k}$ and $\sigma_{i, n-k}$, showed significant differences in $T_{x}, T_{y}$ and $R_{z}(p<0.05)$. Where treatment setup was based on the TBF alone, significant differences in $\Sigma$ and $\sigma^{\prime}$ were only observed in the prostate rotations, whereas $\Sigma$ were statistically larger than the corresponding $\sigma^{\prime}$ in both prostate translation and rotations if online adaptive corrections were applied (Table 1), i.e., the variation of the average position in the pre-treatment CBCT scan relative to the panning CT was larger than the variation within the pre-treatment scans.
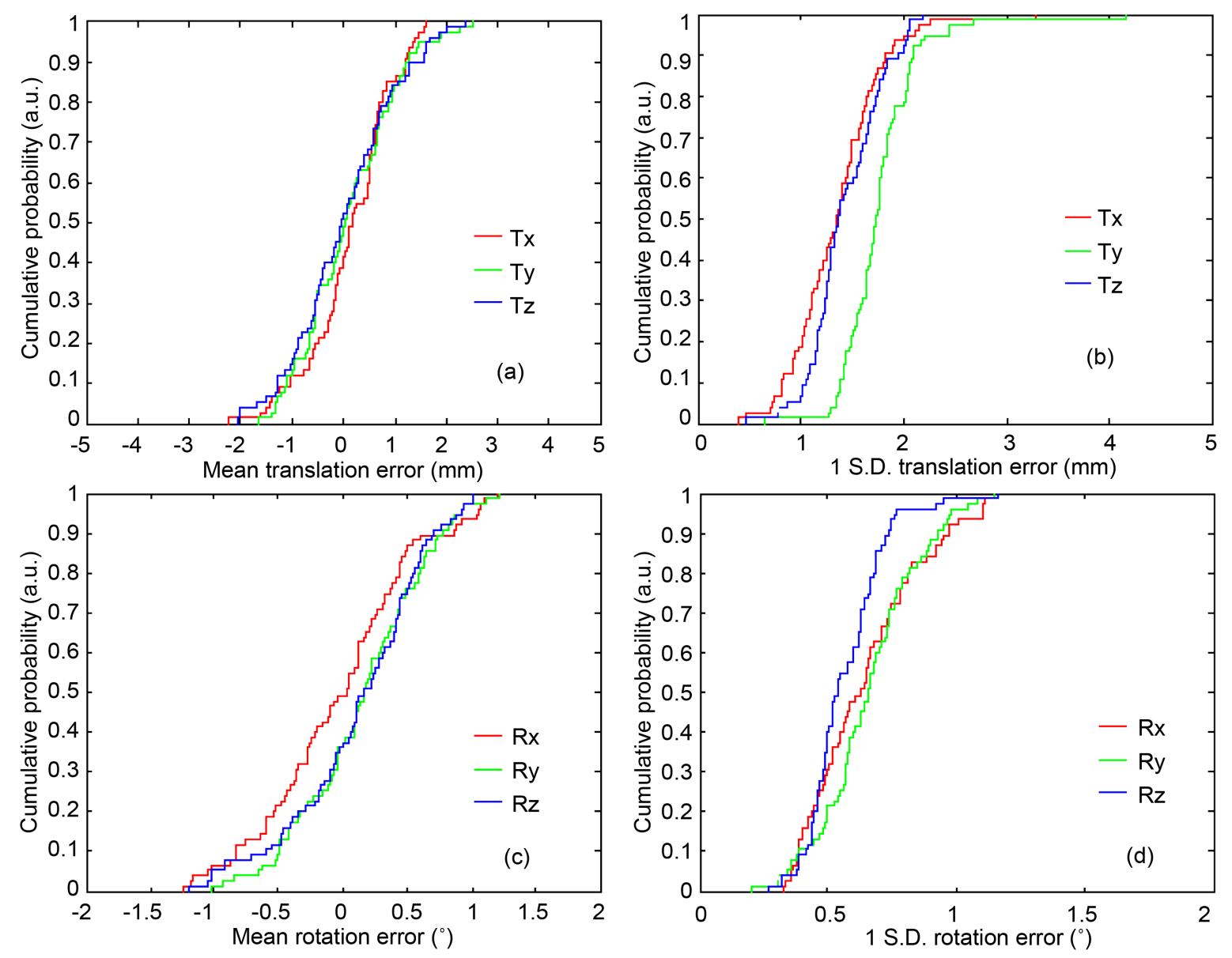

Figure 3. Cumulative probability curve of (a) mean translation error; (b) standard deviation of translation error; (c) mean rotational error; (d) standard deviation of rotational error. 
Table 1. Prostate translation and rotation errors split into overall means (GM), systematic $(\Sigma)$ and random $\left(\sigma^{\prime}\right)$ variations by setup strategies and treatment fractions.

\begin{tabular}{|c|c|c|c|c|c|c|}
\hline \multicolumn{7}{|c|}{ TBF without image-guidance (total fractions) } \\
\hline Translation/rotation axis & $T_{x}$ & $T_{y}$ & $T_{z}$ & $R_{x}$ & $R_{y}$ & $R_{\mathrm{z}}$ \\
\hline $\mathrm{GM}\left(\mathrm{mm} /{ }^{\circ}\right)$ & 0.7 & 0.7 & 0.5 & 0.0 & 0.2 & 0.2 \\
\hline$\Sigma\left(\mathrm{mm} /{ }^{\circ}\right)$ & 1.8 & 2.2 & 1.7 & 0.8 & 0.7 & 0.7 \\
\hline$\sigma^{\prime}\left(\mathrm{mm} /{ }^{\circ}\right)$ & 1.5 & 1.8 & 1.4 & 0.6 & 0.7 & 0.5 \\
\hline PTV (mm) & 6.3 & 7.7 & 5.8 & & & \\
\hline \multicolumn{7}{|c|}{ TBF without image-guidance (first 5 fractions) } \\
\hline Translation/rotation axis & $T_{x}$ & $T_{y}$ & $T_{z}$ & $R_{x}$ & $R_{y}$ & $R_{\mathrm{z}}$ \\
\hline $\mathrm{GM}\left(\mathrm{mm} /{ }^{\circ}\right)$ & 0.8 & 0.8 & 0.5 & 0.0 & 0.2 & 0.2 \\
\hline$\Sigma\left(\mathrm{mm} /{ }^{\circ}\right)$ & 1.7 & 2.1 & 1.7 & 0.6 & 0.5 & 0.5 \\
\hline$\sigma^{\prime}\left(\mathrm{mm} /{ }^{\circ}\right)$ & 1.5 & 1.9 & 1.5 & 0.7 & 0.7 & 0.6 \\
\hline PTV (mm) & 5.8 & 7.3 & 5.8 & & & \\
\hline \multicolumn{7}{|c|}{ TBF with online adaptive image-guidance corrections } \\
\hline Translation/rotation axis & $T_{x}$ & $T_{y}$ & $T_{z}$ & $R_{x}$ & $R_{y}$ & $R_{\mathrm{z}}$ \\
\hline $\mathrm{GM}\left(\mathrm{mm} /{ }^{\circ}\right)$ & 0.1 & 0.1 & 0.0 & 0.0 & 0.2 & 0.1 \\
\hline$\Sigma\left(\mathrm{mm} /{ }^{\circ}\right)$ & 0.8 & 0.9 & 1.0 & 0.6 & 0.5 & 0.5 \\
\hline$\sigma^{\prime}\left(\mathrm{mm} /{ }^{\circ}\right)$ & 1.4 & 1.8 & 1.5 & 0.7 & 0.7 & 0.6 \\
\hline PTV (mm) & 3.3 & 3.8 & 3.8 & & & \\
\hline
\end{tabular}

Treatment safety margins necessary to compensate for the translation errors if using the TBF alone, and if combining online adaptive correction for $\mu_{i, k}$ with the TBF are also given in Table 1 . With the simple strategy of adapting $\mu_{i, k}$ for treatments that followed the first 5 fractions, the margin could have been reduced by 8 (LR), 7 (CC), 7 (anterior) and 5 (posterior) mm comparing to our institution's empirical approach, and by 4 (LR), 5 (CC) and 3 (AP) mm comparing to using the TBF alone.

\subsection{Planning Dosimetry with the Calculated PTV}

Table 2 shows the dosimetric results of 5 other randomly selected patients who were re-planned with the new PTV margins estimated for different TBF-based setup correction strategies. VMAT plans generated with our standard margin, and a $2 \mathrm{~mm}$ margin assuming daily correction for translation prostate errors were included for comparisons. For non-IG setup using the TBF alone, plans obtained with the calculated population-based PTV and our standard protocol showed marginally significant difference in the bladder $\mathrm{V}_{65}$ only $(p=0.043)$. The same results were obtained comparing plans assuming daily IG and adaptive online IG in combination with the TBF, with the dosimetric difference being significant only for the bladder $\mathrm{V}_{65}(p=0.043)$.

The use of IG set a clear difference in the OARs' doses between plans independent of the TBF. For the bladder, $\mathrm{V}_{65 \mathrm{~Gy}}$ and $\mathrm{V}_{40 \mathrm{~Gy}}$ decreased by a large amount of $12.0 \%$ and $12.7 \%$ for the daily IG plans, and by $11.1 \%$ and $12.5 \%$ for the online adaptive plans relative to the standard PTV plans. Due to the smaller PTV overlap with the rectum and the larger space in between, $\mathrm{V}_{65 \mathrm{~Gy}}$ and $\mathrm{V}_{40 \mathrm{~Gy}}$ of the rectum reduced substantially by $6.4 \%$ and $8.5 \%$ for the daily IG plans, and $5.5 \%$ and $6.4 \%$ for the online adaptive plans compared with the standard margin plan. The rectum generalized equivalent uniform dose (gEUD) and the normal tissue complication probability (NTCP) of chronic rectal bleeding estimated by the Lyman model parameters from Ref. [25] were also given in Table 2. Decreased doses to the femoral heads were also observed but not as substantial as to rectum and bladder, and therefore not reported. Validation results from further plan robustness analysis found that the minimum GTV dose $\left(D_{\min }\right)$ was less than the prescription dose of 76 Gy in 3 out of 5 patients, and the GTV volume covered by 
Table 2. Dosimetric metrics of the prostate tumor with $(n=4)$ and without $(n=1)$ seminal vesicle, and selected organs at risk for different setup correction strategies.

\begin{tabular}{|c|c|c|c|c|}
\hline & TBF with daily IG & $\begin{array}{l}\text { TBF with adaptive } \\
\text { online IG }\end{array}$ & $\begin{array}{l}\text { TBF without IG } \\
\text { (calculated PTV) }\end{array}$ & $\begin{array}{l}\text { TBF without IG } \\
\text { (standard PTV) }\end{array}$ \\
\hline \multicolumn{5}{|c|}{ Prostate/without SV } \\
\hline $\mathrm{D}_{\min }(\mathrm{Gy})$ & $76.6(0.5) / 75.6(0.6)$ & $76.5(0.3) / 76.4(0.3)$ & $76.5(0.4) / 76.5(0.2)$ & $76.6(0.2) / 76.3(0.2)$ \\
\hline V76 (\%) & $100(0.0) / 99.0(1.7)$ & $100(0.0) / 99.8(0.2)$ & $100(0.0) / 99.8(0.3)$ & $100(0.0) / 99.7(0.3)$ \\
\hline \multicolumn{5}{|l|}{$\underline{\text { Rectum }}$} \\
\hline V65 (\%) & $11.2(4.7) / 11.6(4.9)$ & $12.1(2.4) / 12.3(2.5)$ & $16.8(2.5) / 17.9(3.1)$ & $17.6(2.4) / 16.9(3.0)$ \\
\hline V40 (\%) & $31.7(4.1) / 32.2(4.2)$ & $33.8(5.7) / 34.7$ (5.8) & $38.3(5.1) / 41.1(3.9)$ & $40.2(3.4) / 39.0(5.4)$ \\
\hline gEUD (Gy) & $60.9(2.4)$ & $61.8(1.1)$ & $64.6(1.1)$ & $64.4(1.1)$ \\
\hline NTCP (\%) & $7.5(2.7)$ & $8.5(1.4)$ & $12.8(2.0)$ & 12.7 (1.9) \\
\hline \multicolumn{5}{|l|}{$\underline{\text { Bladder }}$} \\
\hline V65 (\%) & $8.7(1.9) / 8.5(1.8)$ & $9.6(2.4) / 9.2(2.4)$ & $17.7(3.4) / 20.6(3.1)$ & $20.7(3.2) / 17.2(3.5)$ \\
\hline V40 (\%) & $28.8(11.7) / 28.7(11.7)$ & $29.0(10.2) / 28.8(10.0)$ & $39.6(10.1) / 41.2(9.5)$ & 41.5 (9.5)/39.3 (9.9) \\
\hline
\end{tabular}

76 Gy (V76) was, on average, 99.0\% if combining daily IG with the TBF immobilization while using 2 mm margin to account for the residual rotation errors (Table 2). For other correction strategies with the TBF immobilization, the averaged GTV $\mathrm{D}_{\min }$ and V76 were above $76 \mathrm{~Gy}$ and nearly $100 \%$, respectively. The residual translation and rotation errors have clinically negligible impacts on the OARs' doses regardless of the setup correction strategies as V65 and V40 of the bladder and rectum with and without simulation of residual errors differed by $-2.7 \%$ to $0.9 \%$. In two non-IG plans achieved with the calculated population-based PTV, the rectum V65 simulated for residual errors exceeds the constraint of $20 \%$ slightly by $1.2 \%$.

\section{Discussion}

Effective immobilization and positioning system is important to reduce the interfractional setup variability in radical radiotherapy of prostate cancer, particularly for the patient's rotation which cannot be completely corrected with standard treatment couch. For some ultrasound image-guidance systems that provide a solution of translation shift correction only, it becomes utterly important to limit the prostate rotation in avoidance of potentially serious tumor underdosing. This study is one of the few that focused on the effect of the immobilization system on the prostate translation as well as rotation from its planned position.

\subsection{Rotations of the Prostate}

Using a contour-based approach to estimate the prostate rotation from the analysis of 2679 pretreatment CBCT, it was suggested that our TBF immobilization was able to limit the pitch $\left(R_{x}\right)$, roll $\left(R_{y}\right)$ and yaw $\left(R_{z}\right)$ to within $1^{\circ}$ (group mean), and maximum individual mean error $R_{x}$ less than $2.5^{\circ}$ in a cohort of 80 patients. The preparation and delivery errors in terms of systematic and random errors were equal for $R_{x}$ error $\left(0.6^{\circ}\right)$, which were of similar magnitude for the $R_{y}$ and $R_{z}$ errors $\left(0.5^{\circ}\right)$. In comparisons, large systematic and random $R_{x}$ errors up to $5.1^{\circ}$ $10.1^{\circ}$ and $3.6^{\circ}-7.7^{\circ}$ were observed from repeated CT with patients immobilized by the standard knee support without further fixation over the hips [23], [26]. Large systematic and random $R_{x}$ errors in the range of $2.7^{\circ}-$ $7.6^{\circ}$ and $2.5^{\circ}-10.2^{\circ}$ were also observed from fiducial markers-based studies using similar standard immobilization approaches[8], [26]. Besides the immobilization method, other factors that possibly contributed to the disparity of measured prostate rotations between this study and others include differences of registration method, imaging modality and the reference rotation frame (skin marks or bony landmarks). Notably, the prostate rotation varied with the focus of registration as Owen et al. [26] demonstrated that CBCT registration targeting at the apex, the base or the overall prostate contour could result in the prostate rotating more or less anteriorly or posteriorly. Our institution does not have a rigid guideline for the rotation alignment of the prostate and seminal 
vesicles. For the 18 radiotherapists involved in the setup procedure, most of them barely ever adjusted the rotations from the auto-registration results but all of them did inspect the prostate rotation carefully in the three major planes. On the other hand, Hoogeman et al. [23] reported that the systematic rotation error reduced by a factor of 2 based on the adaptive online correction using the planning CT and 4 pre-treatment CBCT. In Table 1, the systematic errors calculated from the first 5 fractions were about $25 \%$ larger than those calculated from the remainder of $n-5$ fractions after adaptive online correction but the absolute reduction was only $0.2^{\circ}$.

\subsection{Translation of the Prostate}

This study did not consider separately the translational shifts of the bony landmarks and the prostate gland. Using the TBF alone, we found that systematic errors are generally smaller in the LR $(0.6$ - $1.5 \mathrm{~mm})$ and AP (1.3 - 3.2 $\mathrm{mm})$ directions but larger in the SI $(\leq 0.5 \mathrm{~mm})$ directions compared to other similar studies. For example, systematic and random errors in the LR, SI and AP directions were reported to be 2.3, 1.7, and $4.3 \mathrm{~mm}$ and 3.2, 2.3, and $3.9 \mathrm{~mm}$ by Wong et al. [27], and 2.4, 2.7, and $3.0 \mathrm{~mm}$ and 2.5, 2.2, and $3.2 \mathrm{~mm}$ by Mayyas et al. [28] using a leg rest as minimal immobilization, and 2.6, 1.6, $4.9 \mathrm{~mm}$ (systematic) and 2.8, 4.7, and $4.5 \mathrm{~mm}$ (random) by Bylund et al. [6] using a deformable Vac-Lok system. Melancon et al. [20] found smaller systematic errors of 1.0 and $3.6 \mathrm{~mm}$ in a patient group immobilized from the upper thighs to the feet by the Vac-Lok system versus 3.2 and $4.1 \mathrm{~mm}$ with the simple dual leg positioner device in the LR and AP directions while the random errors were similar in all directions. Unlike other studies that showed larger random errors, systematic and random errors are numerically similar in our patient cohort, suggesting that the variation of the average prostate position in the CBCT scans with respect to the planning situation is similar to the variation within the CBCT scans.

Although the TBF immobilization can effectively limit the systematic errors, incidental prostate displacement due to mis-recorded setup with the TBF is not impossible entirely, and was exposed in one patient from this study. This type of error could happen in setup based on the skin marks as reported in Wong et al. [27] showing a lateral prostate displacement up to $27 \mathrm{~mm}$. This highlighted the importance of some level of IG correction albeit the effectiveness of the TBF immobilization overall.

The advantage of combining our TBF with adaptive offline IG correction was obvious (Figure 3), as the individual systematic translations can be corrected early into the treatment. Our result that showed significant differences in systematic for individual patients between the first five fractions and the remaining fractions after online adaptive correction is consistent with Snir et al. where the statistical significance were found to vanish by the tenth fractions [7]. The resulting population systematic translation were also found to decrease by $0.9,1.2$, and $0.7 \mathrm{~mm}$ in the LR CC and AP directions relative to using the TBF alone without any IG.

\subsection{PTV Margin and Dosimetric Impacts}

Because the uncertainty in prostate position in the planning CT determines the systematic variation, our standard PTV of $10 \mathrm{~mm}$ can be decreased by 3 - $4 \mathrm{~mm}$ in the LR and SI directions but the posterior $5 \mathrm{~mm}$ margin was 1 $\mathrm{mm}$ short if patients were treated solely based on the TBF setup without image guidance applied. At a combined error of $5 \mathrm{~mm}$ assuming the random contribution of $1.5 \mathrm{~mm}$ in Table 1, individual systematic error $\geq 1.4 \mathrm{~mm}$ was relatively frequent (in 10 out of 80 patients) and therefore $5 \mathrm{~mm}$ margin may be inadequate if asymmetric margin calculation was considered. Since dose to rectum is mainly determined by the PTV size at the posterior, treatment plans achieved with the standard PTV and the calculated PTV do not show significant differences in the rectum dose constraint parameters and the estimated complication rates in such TBF only setup scenario.

The theoretical benefit of daily IG using CBCT has long been a debatable issue because it increases not just the workload but more importantly the imaging dose and potentially risk of secondary cancers. Nonetheless, with daily CBCT to have the translation prostate error corrected and the rotations limited to within $2^{\circ}$ by the TBF, the PTV can be made to very small. The arbitrary $2 \mathrm{~mm}$ assumed in this study was found to be sufficient to protect the prostate and seminal vesicle from being under-dosed in our plan robustness analysis based on the realistic prostate rotations of five random sample patients. The dependency of rectal dose and the PTV size has been investigated in a few studies [29], [30]. Our results also confirmed that both dosimetric gains and rectum toxicity can be significantly improved relative to the non-IG treatment approach despite the use of an effective immobilization device like our TBF.

On the other hand, the plan dose statistics achieved with the adaptive online correction were found to be similar to those of the daily IG plans and similarly adequate in the presence of modeled uncertainty in target posi- 
tion. Note that the prostate rotation errors were not accounted for by the van Herk's margin recipe but were encompassed by our calculated margin for the adaptive correction strategy as the prostate seminal vesicle was found to attain $99 \%$ dose coverage on average. This could be explained by the smaller systematic errors of individual patients than used in the margin calculation based on the population statistics. The same finding was obtained for the scenario of the TBF only setup. A limitation of this study is that the effect of the TBF immobilization on the intrafractional variability of prostate position was not evaluated, and hence the calculated PTV did not reflect the actual PTV.

Reducing the PTV is always a difficult decision within many institutions, although evidences from a number of prostate motion analysis and setup evaluations constantly indicated the feasibility through daily or adaptive online IG. This can be understood by the past experiences that showed acceptable outcomes with the empirical margin. In one study comparing two groups of patients treated by IMRT with and without daily CBCT imageguidance, at the same margin budget Zhong et al. [31] found no significant improvement in biochemical control at 5 years and difference in gastrointestinal and genitourinary toxicities. Still, there have been a growing number of clinical studies that showed comparable biochemical failure rates with individualized or treatment-specific PTV, which supports a change of margin practice to ensue lower normal tissue toxicities [32]. Further follow-up investigation of the clinical outcomes is warranted to ensure the appropriateness of our IG TBF-based setup and to provide useful information in determining the optimal margin in our next-stage real-time ultrasound-monitoring hypofractionated radiotherapy of localized prostatic tumors.

\section{Conclusion}

Combining adaptive online correction or daily CBCT image-guidance with a total body immobilization and positioning device can effectively limit the prostate translation and rotation errors, which is particularly important in delivering high dose conventional fractionated and hypofractionated radiotherapy with standard treatment couch and using image-guidance system without rotation solution of correction.

\section{References}

[1] Beckendorf, V., Guerif, S., Le Prisé, E., Cosset, J.-M., Bougnoux, A., Chauvet, B., et al. (2011) 70 Gy versus 80 Gy in Localized Prostate Cancer: 5-Year Results of GETUG 06 Randomized Trial. International Journal of Radiation Oncology*Biology*Physics, 80, 1056-1063. http://dx.doi.org/10.1016/j.ijrobp.2010.03.049

[2] Arcangeli, G., Saracino, B., Gomellini, S., Petrongari, M.G., Arcangeli, S., Sentinelli, S., et al. (2010) A Prospective Phase III Randomized Trial of Hypofractionation versus Conventional Fractionation in Patients with High-Risk Prostate Cancer. International Journal of Radiation Oncology*Biology*Physics, 78, 11-18. http://dx.doi.org/10.1016/j.ijrobp.2009.07.1691

[3] Sanpaolo, P., Barbieri, V. and Genovesi, D. (2014) Biologically Effective Dose and Definitive Radiation Treatment for Localized Prostate Cancer. Strahlentherapie und Onkologie, 190, 732-738. http://dx.doi.org/10.1007/s00066-014-0642-0

[4] Madsen, B.L., His, R.A., Pham, H.T., Fowler, J.F., Esagui, L. and Corman, J. (2007) Stereotactic Hypofractionated Accurate Radiotherapy of the Prostate (SHARP), 33.5 Gy in Five Fractions for Localized Disease: First Clinical Trial Results. International Journal of Radiation Oncology*Biology*Physics, 67, 1099-1105. http://dx.doi.org/10.1016/j.ijrobp.2006.10.050

[5] Leborgne, F. and Fowler, J. (2008) Acute Toxicity after Hypofractionated Conformal Radiotherapy for Localized Prostate Cancer: Nonrandomized Contemporary Comparison with Standard Fractionation. International Journal of Radiation Oncology*Biology*Physics, 72, 770-776.e1. http://dx.doi.org/10.1016/j.ijrobp.2008.01.023

[6] Bylund, K.C., Bayouth, J.E., Smith, M.C., Hass, A.C., Bhatia, S.K. and Buatti, J.M. (2008) Analysis of Interfraction Prostate Motion Using Megavoltage Cone Beam Computed Tomography. International Journal of Radiation Oncology*Biology*Physics, 72, 949-956. http://dx.doi.org/10.1016/j.ijrobp.2008.07.002

[7] Snir, J.A., Battista, J.J., Bauman, G. and Yartsev, S. (2011) Evaluation of Inter-Fraction Prostate Motion Using Kilovoltage Cone Beam Computed Tomography during Radiotherapy. Clinical Oncology, 23, 625-631.

http://dx.doi.org/10.1016/j.clon.2011.03.007

[8] Graf, R., Boehmer, D., Budach, V. and Wust, P. (2012) Interfraction Rotation of the Prostate as Evaluated by Kilovoltage X-Ray Fiducial Marker Imaging in Intensity-Modulated Radiotherapy of Localized Prostate Cancer. Medical Dosimetry, 37, 396-400. http://dx.doi.org/10.1016/j.meddos.2012.02.006

[9] van de Water, S., Valli, L., Aluwini, S., Lanconelli, N., Heijmen, B. and Hoogeman, M. (2014) Intrafraction Prostate 
Translations and Rotations during Hypofractionated Robotic Radiation Surgery: Dosimetric Impact of Correction Strategies and Margins. International Journal of Radiation Oncology*Biology*Physics, 88, 1154-1160. http://dx.doi.org/10.1016/j.ijrobp.2013.12.045

[10] Lin, Y., Liu, T., Yang, W., Yang, X. and Khan, M.K. (2013) The Non-Gaussian Nature of Prostate Motion Based on Real-Time Intrafraction Tracking. International Journal of Radiation Oncology*Biology*Physics, 87, 363-369. http://dx.doi.org/10.1016/j.ijrobp.2013.05.019

[11] Bohrer, M., Schröder, P., Welzel, G., Wertz, H., Lohr, F., Wenz, F., et al. (2008) Reduced Rectal Toxicity with Ultrasound-Based Image Guided Radiotherapy Using BAT ${ }^{\mathrm{TM}}$ (B-Mode Acquisition and Targeting System) for Prostate Cancer. Strahlentherapie und Onkologie, 184, 674-678. http://dx.doi.org/10.1007/s00066-008-1837-z

[12] Ricardi, U., Franco, P., Munoz, F., Levis, M., Fiandra, C., Guarneri, A., et al. (2015) Three-Dimensional UltrasoundBased Image-Guided Hypofractionated Radiotherapy for Intermediate-Risk Prostate Cancer: Results of a Consecutive Case Series. Cancer Investigation, 33, 23-28. http://dx.doi.org/10.3109/07357907.2014.988343

[13] Rijkhorst, E.-J., van Herk, M., Lebesque, J.V. and Sonke, J.-J. (2007) Strategy for Online Correction of Rotational Organ Motion for Intensity-Modulated Radiotherapy of Prostate Cancer. International Journal of Radiation Oncology*Biology*Physics, 69, 1608-1617. http://dx.doi.org/10.1016/j.ijrobp.2007.08.042

[14] Yue, N.J., Knisely, J.P.S., Song, H. and Nath, R. (2005) A Method to Implement Full Six-Degree Target Shift Corrections for Rigid Body in Image-Guided Radiotherapy. Medical Physics, 33, 21-31. http://dx.doi.org/10.1118/1.2138009

[15] Deutschmann, H., Kametriser, G., Steininger, P., Scherer, P., Schöller, H., Gaisberger, C., et al. (2012) First Clinical Release of an Online, Adaptive, Aperture-Based Image-Guided Radiotherapy Strategy in Intensity-Modulated Radiotherapy to Correct for Inter- and Intrafractional Rotations of the Prostate. International Journal of Radiation Oncology*Biology*Physics, 83, 1624-1632. http://dx.doi.org/10.1016/j.ijrobp.2011.10.009

[16] Lips, I.M., van der Heide, U.A., Kotte, A.N.T.J., van Vulpen, M. and Bel, A. (2009) Effect of Translational and Rotational Errors on Complex Dose Distributions with Off-Line and On-Line Position Verification. International Journal of Radiation Oncology, Biology, Physics, 74, 1600-1608. http://dx.doi.org/10.1016/j.ijrobp.2009.02.056

[17] Shang, Q., Sheplan Olsen, L.J., Stephans, K., Tendulkar, R. and Xia, P. (2013) Prostate Rotation Detected from Implanted Markers Can Affect Dose Coverage and Cannot Be Simply Dismissed. Journal of Applied Clinical Medical Physic, 14, 177-191.

[18] White, P., Yee, C., Shan, L., Chung, L., Man, N. and Cheung, Y. (2014) A Comparison of Two Systems of Patient Immobilization for Prostate Radiotherapy. Radiation Oncology, 9, 29. http://dx.doi.org/10.1186/1748-717X-9-29

[19] Malone, S., Szanto, J., Perry, G., Gerig, L., Manion, S., Dahrouge, S., et al. (2000) A Prospective Comparison of Three Systems of Patient Immobilization for Prostate Radiotherapy. International Journal of Radiation Oncology, Biology, Physics, 48, 657-665. http://dx.doi.org/10.1016/S0360-3016(00)00682-9

[20] Melanccon, A.D., Kudchadker, R.J., Amos, R., Johanson, J., Zhang, Y., Yu, Z.H., et al. (2013) Patient-Specific and Generic Immobilization Devices for Prostate Radiotherapy. International Journal of Medical Physics, Clinical Engineering and Radiation Oncology, 2, 125-132. http://dx.doi.org/10.4236/ijmpcero.2013.24017

[21] Lee, E., Yuen, K., Mui, W., Law, G.M.L., Lui, C., Chan, M.K.H., et al. (2013) Salvage Radiotherapy to the Prostatic Fossa Using Volumetric-Modulated Arc Therapy: Early Results. Hong Kong Journal of Radiology, 16, 191-197. http://dx.doi.org/10.12809/hkjr1313173

[22] Van Herk, M. (2004) Errors and Margins in Radiotherapy. Seminars in Radiation Oncology, 14, 52-64. http://dx.doi.org/10.1053/j.semradonc.2003.10.003

[23] Hoogeman, M.S., van Herk, M., de Bois, J. and Lebesque, J.V. (2005) Strategies to Reduce the Systematic Error Due to Tumor and Rectum Motion in Radiotherapy of Prostate Cancer. Radiotherapy and Oncology, 74, 177-185. http://dx.doi.org/10.1016/j.radonc.2004.12.010

[24] Deasy, J.O., Blanco, A.I. and Clark, V.H. (2003) CERR: A Computational Environment for Radiotherapy Research. Medical Physics, 30, 979-985. http://dx.doi.org/10.1118/1.1568978

[25] Söhn, M., Yan, D., Liang, J., Meldolesi, E., Vargas, C. and Alber, M. (2007) Incidence of Late Rectal Bleeding in High-Dose Conformal Radiotherapy of Prostate Cancer Using Equivalent Uniform Dose-Based and Dose-VolumeBased NTCP Models. International Journal of Radiation Oncology, Biology, Physics, 67, 1066-1073. http://dx.doi.org/10.1016/j.ijrobp.2006.10.014

[26] Owen, R., Kron, T., Foroudi, F., Milner, A., Cox, J. and Duchesne, G. (2011) Interfraction Prostate Rotation Determined from In-Room Computerized Tomography Images. Medical Dosimetry, 36, 188-194. http://dx.doi.org/10.1016/j.meddos.2010.03.002

[27] Wong, J.R., Gao, Z., Uematsu, M., Merrick, S., Machernis, N.P., Chen, T., et al. (2008) Interfractional Prostate Shifts: Review of 1870 Computed Tomography (CT) Scans Obtained during Image-Guided Radiotherapy Using Ct-on-Rails for the Treatment of Prostate Cancer. International Journal of Radiation Oncology, Biology, Physics, 72, 1396-1401. 
http://dx.doi.org/10.1016/j.ijrobp.2008.03.045

[28] Mayyas, E., Chetty, I.J., Chetvertkov, M., Wen, N., Neicu, T., Nurushev, T., et al. (2013) Evaluation of Multiple Image-Based Modalities for Image-Guided Radiation Therapy (IGRT) of Prostate Carcinoma: A Prospective Study. Medical Physics, 40, Article ID: 041707. http://dx.doi.org/10.1118/1.4794502

[29] Langsenlehner, T., Döller, C., Winkler, P., Gallé, G. and Kapp, K.S. (2013) Impact of Inter- and Intrafraction Deviations and Residual Set-Up Errors on PTV Margins. Strahlentherapie und Onkologie, 189, 321-328. http://dx.doi.org/10.1007/s00066-012-0303-0

[30] Kukołowicz, P., Kukołowicz, H. and Tyburska, I. (2015) Dependence of the Safe Rectum Dose on the CTV-PTV Margin Size and Treatment Technique. Reports of Practical Oncology and Radiotherapy, 20, 198-203. http://dx.doi.org/10.1016/j.rpor.2014.12.004

[31] Zhong, Q., Gao, H., Li, G., Xiu, X., Wu, Q., Li, M., et al. (2014) Significance of Image Guidance to Clinical Outcomes for Localized Prostate Cancer. BioMed Research International, 2014, Article ID: 860639. http://dx.doi.org/10.1155/2014/860639

[32] Chu, W., Loblaw, D., Chan, K., Morton, G., Choo, R., Szumacher, E., et al. (2015) Long-Term Results of a Study Using Individualized Planning Target Volumes for Hypofractionated Intensity-Modulated Radiotherapy Boost for Prostate Cancer. Radiation Oncology, 10, 95. http://dx.doi.org/10.1186/s13014-015-0400-1

\section{Submit or recommend next manuscript to SCIRP and we will provide best service for you:}

Accepting pre-submission inquiries through Email, Facebook, LinkedIn, Twitter, etc. A wide selection of journals (inclusive of 9 subjects, more than 200 journals)

Providing 24-hour high-quality service

User-friendly online submission system

Fair and swift peer-review system

Efficient typesetting and proofreading procedure

Display of the result of downloads and visits, as well as the number of cited articles

Maximum dissemination of your research work

Submit your manuscript at: http://papersubmission.scirp.org/ 